\title{
Effects of Social Networking by Influencers [Beware and Stay Safe]
}

\author{
P. Adarsha ${ }^{1}$, Dr. N. Chandra Sekhar Reddy ${ }^{2}$, S. Srinivas ${ }^{3}$ \\ ${ }^{I}$ Student, M.Tech IT Dept., Institute of Aeronautical Engineering, HYD-500043, AP, India. \\ ${ }^{2}$ Professor, CSE Dept., Institute of Aeronautical Engineering, HYD-500043, AP, India. \\ ${ }^{3}$ Asst.Professor, IT Dept., Institute of Aeronautical Engineering, HYD-500043, AP, India.
}

\begin{abstract}
A short time agone, social spam and on-line abuse were much non-existent. As email spam filters became simpler, catching over ninety fifth of those messages, spammers have moved to a replacement target the social net. Over ninetieth of social network users have knowledgeable about social spam in some kind.Those doing the "spamming" is automatic spam-bots, fault accounts, or real individuals.. during this paper we have a tendency to investigate strategies for exploiting such community feedback to mechanically determine prime quality content. As a action at law, we have a tendency to specialise in Yahoo! Answers, an oversized community question/answering portal that's significantly made within the quantity and kinds of content and social interactions accessible in it. we have a custom to introduce a general classification framework for merging the proof from totally differential sources of knowledge, which will be tuned automatically for a given social media sort and quality definition. above all, for the community question/answering domain, we have a custom to show that our system is in a position to separate high-quality things from the remainder with accuracy near that of humans.
\end{abstract}

Keywords: social net ,spamming , fault accounts, social media, passwords, intruders attacks

\section{Introduction:}

Social networking could be a tool utilized by individuals all round the globe. Its purpose is to push and aid communication. However, this sort of technology may be doing a lot of damage. it's not solely dynamic however we have a tendency to communicate, we have a tendency to move with one another in standard of living. Sarah Zay, of USA these days, expressed that "With the increase of online sites like Facebook, social networking is also on the verge of substitution ancient personal interactions for succeeding the generations".

Social networks, like Facebook, were created for the main purpose to serve the to people communicate. There are several alternative reasons that these technologies are unit used, however communication will remain the quantity one. many folks use these networks to interact with their friends in alternative cities, states, or maybe alternative countries. There are many things that don't permit the utilization of telephones; this can be why social networking is preferred by several. These networks not solely permit communication between friends, however permit you to fulfill new individuals. Similarities and customary friends will produce new bonds. this permits one's social circle to expand. If you raise around however individuals assume social networking affects them, several can say that they, "Have an enlarged circle of social connections, and keep in more contact".These networks have become the trendy thanks to create friends. Finally, new friends communicate through these networks.

\section{Existing System:}

Existing approaches to Users usually make it easier to remember their passwords, users often use the same or similar passwords on each system; and given a choice, most users will select a very simple and easy-toremember password such as their birthday, their mother's maiden name, or the name of a relative. Short and simple passwords are relatively easy for attackers to determine.dejectedly, these passwords are broken remorselessly by intruders by many easy means like eaves dropping, masquerading, and alternative rude means that say shoulder surfing attacks,social engineering attacks, lexicon attacks.

Disadvantage: 1 .The sturdy system-assigned passwords are troublesome for users to recollect.

\section{Proposed System:}

The main plan behind this proposal is to cut back the guess attacks likewise as encouraging users to pick out additional random, and tough positive identifications to guess by merg persuasive cued click points and password guess resistant protocol. A password authentication system should encourage strong passwords while maintaining memorability.In an attempt to create more memorable passwords, graphical password system have been devised. In these systems authentication is based on clicking on images rather than typing alphanumeric strings[6]. 
Advantage: 1. Human brain is nice in memory image than matter character.

\section{Types In Social Networks :}

There are many varieties of social networks obtainable. This reality sheet examines the privacy and security implications of employing a few of them. Most social networks mix components of quite one among these forms of networks, and also the focus of a social network could amendment over time. whereas this reality sheet doesnl't address each style of social network, several of the protection and privacy recommendations square measure applicable to alternative forms of networks.[2]

Personal networks : These networks enable users to form elaborate on-line profiles and connect with other users, with a stress on social relationships(e.g. Facebook)

Status update networks : These forms of social networks are designed to allow users to post short standing updatess so as to speak with other users fastly (e.g. Twitter)

Location networks: This automatically sets the appropriate firewall and security settings for the type of network that you connect to.

Content-sharing networks. These are designed as platforms for sharing content, like videos, music and pictures.

\section{Networking Activities :}

There is significant interest in social networking in middle and low-income nations. Individuals at high rates get involved consistently once they are online in these countries. In countries like Japan and European half all web users join in social networks. and people Japanese and Germans World Health Organization do go surfing for social networking use it less usually than others round the world to particular thoughts on culture, spots, politics, community and religion[3].Likewise, use of social networking sites differs by education level.

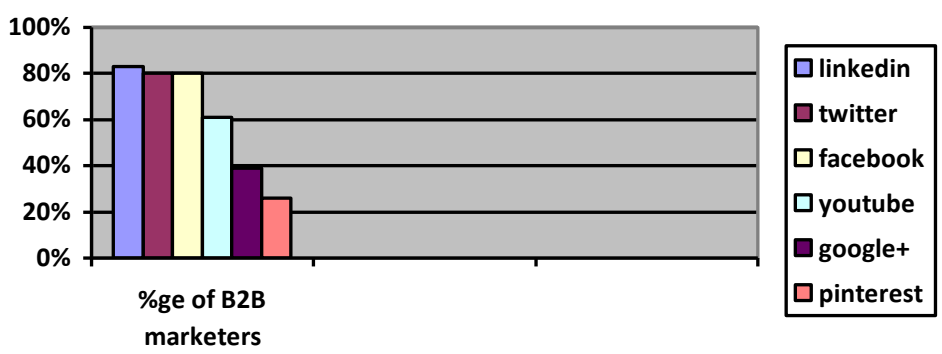

VI. Information Viability :

When posting info to a social network, a user in all probability expects approved contacts to be able to read it. Entities that collect personal info for legal functions include:Advertisers curious about personal info in order that they will higher target their ads to those perhaps to have an interest within the product. Software package developers of Third-Party who includes the information to change applications, such as games in the internet that moves with the Entities of social network which gathers personal information for an outlawed functions include:Identitying the attackers who get personal information either supportive information others post or else that of a user posts concerning the user. different on-line criminals, like individuals attending to scam or harass people, or infect laptops with malware[4]

\section{Bootstrapping The Foaf-Web - The Experiment :}

FOAF is one in every of the foremost used vocabularies, it provides the essential categories and properties necessary to explain the people and their relationships.however, FOAF descriptions may be found just for a really tiny portion of net users. Bootstrapping may be a drawback that influence all applications of the linguistics net, also the network of interlinked Friend-of-a-Friend (FOAF) profiles mentioned to as the FOAFweb. during this paper we tend to introduce a hybrid system that not only gathers existing FOAF professional files from the linguistics net, but additionally applies best-known web-mining techniques to extract social network info from the initial, machine-readable text net. The system additionally lets the users visualize and browse the ensuing network so as to get friends in common, friends thought to be lost or potential new friendships supported shared interests. 


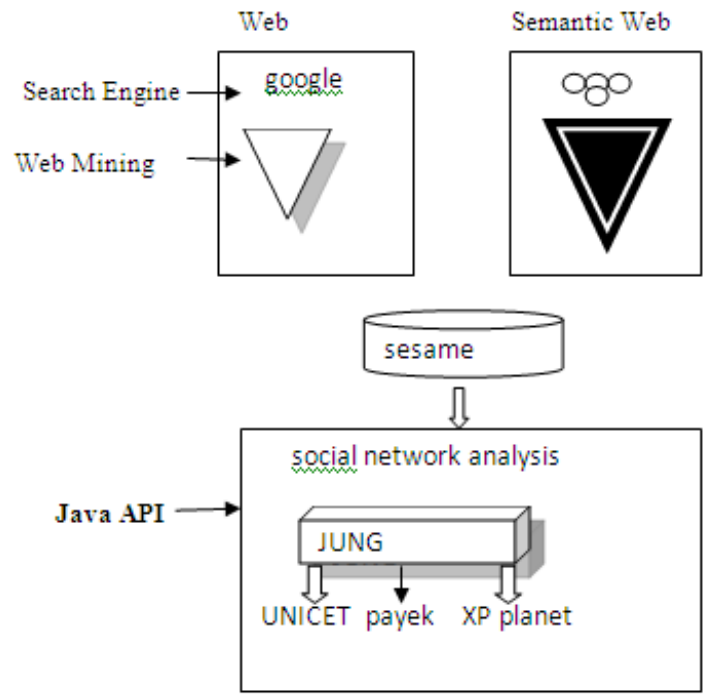

\section{Action Graph:}

an action graph for action $\mathrm{a}, \mathrm{Ga}=(\mathrm{Ua} ; \mathrm{Ea})$ consists of a group of Users Ua who have performed action a at some purpose of your time and set of directed edges (ui; uj) such an action was propagated from node ui to uj .

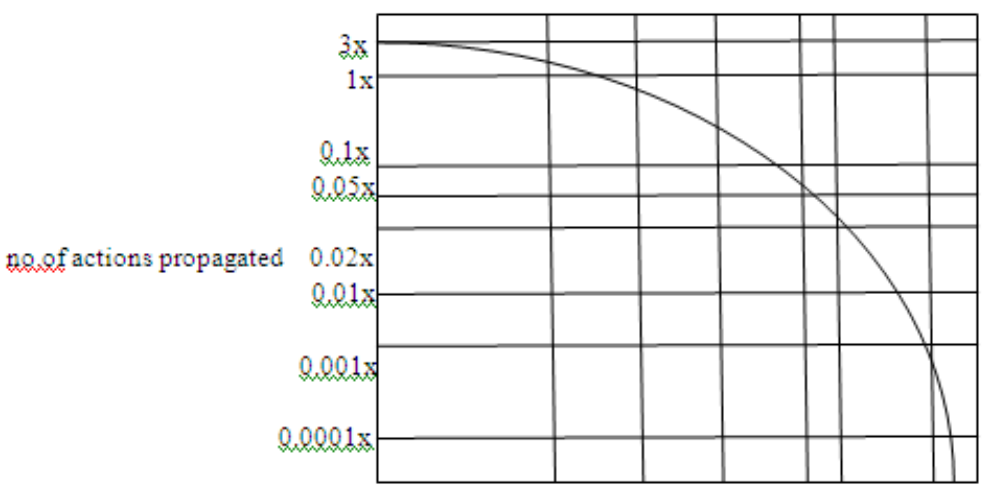

\section{Algorithm one Computing the Action Graph}

Step 1: Take Input of $\mathrm{C}$ as (ui; $\mathrm{uj})$ and $\mathrm{A}$ as (u; a; tau)

Step 2: we tend to get Output for $\mathrm{Ga}$ as $(\mathrm{Ua} ; \mathrm{Ea})$

Step 3: for every tuple (ui; uj) in C do

Step 4: for every action event (u; a; tau) in a do

Step 5: if A contains entries for ui\&uj then

Step 6: if (taui $\square$ tauj) $<=$ then

Step 7: ui+uj = Ua;

Step 8: add a directed edge ((ui; uj)) to Ea;

Step 9: else if (tauj $\square$ taui) $<=$ then

Step 10: ui+uj=Ua;

Step 11: add a directed edge ((uj;ui)) to Ea;

Step 12: end if

Step 13: end if

Step 14: end for

Step 15: end for

\section{Experimental Evaluation:}

Based on our analysis within the previous section, we have a tendency to propose a solutionswer $\}$ to the matter exhibit of predicting the reach price for an action and a user. during this section, we have a tendency to describe the traintest split, model and options used. 


\section{Training and Testing}

From the information, wel've got a user-action combine and also the determined reacha(u) price. we have a tendency to cipher the $\log$ of the reacha(u) and use it because the reach price. for every user-action combine the goal is to predict the reach of the cascade, as if we have a tendency to failed to realize the cascade event. every entry in our dataset consists of the subsequent tuple, $(\mathrm{u}, \mathrm{a}, \mathrm{F}, \log (\operatorname{reacha}(\mathrm{u})))$. wherever $\mathrm{F}$ is that the set of options delineated earlier. All the feature values in $\mathrm{F}$ are computed on the action log designed until timeM.We use the information from timeM +1 forwards for the experiments. the concept is to be told from the past user and action behaviors to effectively predict the reach in future for a user action combine. In our case a user solely performs an action once, hence we have a tendency to take a look at the model on ( $u$; a) specified $u$ failed to perform action a earlier in time M. In cases, wherever a user will perform a similar action quite once (for e.g. clicking on an ad), the model may be accustomed predict the reach for a similar user-action combine. we have a tendency to split our information into magnitude relation 60:30:10 for training, testing and validation severally. we have a tendency to make sure that these sets are non-overlapping w.r.t the actions, that is, all the tuples $(\mathrm{u}, \mathrm{a}, \mathrm{F}, \log ($ reacha( $(\mathrm{u})))$ having action a can come in either of the coaching, take a look at or validation set. As our goal is to predict a true valued range (reacha(u)), we have a tendency to solid it as a regression downside. we have a tendency to use Gradient boosted call trees (GBDT) as a regression model for predicting the reach values.The GBDT parameters, range of trees and range of leaf nodes per tree were set to one hundred fifty and a hundred severally.

\section{Conclusion :}

The outlook of related work is distant and vast due to the interdisciplinary nature of work, merging Web Intelligence and Social Network Analysis. regarding our experiment, the closest precursor to be found in the literature is the Referral Web project, where data about the AI community in a same fashion by crawling the web and exploiting a search engine was collected.Still, data was used for a different purpose, namely interactive exploration of the network and the automation of referrals .By analyzing the linking structure of the Web (eg: the EICSTES project) or relying on publication or project databases traditionally scientific communities have also been studied. Related to our method, for the purposes of ontology learning Google has been used most recently by Cimiano et al.

\section{References:}

[1]. Hila Becker_y, Dan Itery,"Identifying Content for Planned Events Across Social Media Sites"Proceedings of the National Academy of Sciences, 105(41):15649\{15653, 2008.

[2]. D. A. Shamma, L. Kennedy, and E. Churchill. Statler: Summarizing media through short-message services. In Proceedings of the 2010 ACM Conference on Computer Supported Cooperative Work (CSCW '10), 2010.

[3]. M. Naaman, H. Becker, and L. Gravano. Hip and trendy: Characterizing emerging trends on Twitter. Journal of the American Society for Information Science and Technology,62(5):902\{918, 2011.

[4]. L. Liu, L. Sun, Y. Rui, Y. Shi, and S. Yang. Web video topic discovery and tracking via bipartite graph reinforcement model. In Proceedings of the 17th International World Wide Web Conference (WWW '08), 2008.

[5]. H. Kwak, C. Lee, H. Park, and S. Moon. What is Twitter, a social network or a news media? In Proceedings of the19 ${ }^{\text {th }}$ International World Wide Web Conference (WWW '10), 2010.

[6]. Susan Wiedenbeck Jim Waters, Jean-Carnille Birget, Alex Brodskiy Nasir Memon. SOUPS` 05, July 6-8, 2005, Pittsburg, PA, USA. 